\title{
Magnetic moments of the spin-1/2 singly charmed baryons in covariant baryon chiral perturbation theory
}

\author{
Rui-Xiang Shi, Yang Xiao, and Li-Sheng Geng* \\ School of Physics and Nuclear Energy Engineering \& International Research Center for Nuclei and \\ Particles in the Cosmos \& Beijing Key Laboratory of Advanced Nuclear Materials and Physics, \\ Beihang University, Beijing 100191, China
}

(Received 24 December 2018; published 17 September 2019)

\begin{abstract}
Recent experimental advances have reignited theoretical interests in heavy-flavor hadrons. In this work, we study the magnetic moments of the spin-1/2 singly charmed baryons up to the next-to-leading order in covariant baryon chiral perturbation theory (BChPT) with the extended-on-mass-shell renormalization (EOMS) scheme. The pertinent low energy constants (LECs) $g_{1-4}$ are fixed with the help of the quark model and the heavy quark spin flavor symmetry, while the remaining $d_{2}, d_{3}, d_{5}$, and $d_{6}$ are determined by fitting to the lattice QCD pion-mass dependent data. With the LECs so determined, we predict the magnetic moments of the spin- $1 / 2$ singly charmed baryons and compare them with those of other approaches, and find that our predictions are in general smaller in absolute values than those of other approaches, which are tied to the lattice QCD data we fitted. More studies are therefore needed to clarify this situation and to better understand the nature of the singly charmed baryons.
\end{abstract}

DOI: 10.1103/PhysRevD.100.054019

\section{INTRODUCTION}

In the last two decades, tremendous progress has been made in our understanding of heavy-flavor hadrons, thanks to the experimental discoveries by collaborations such as LHCb, BELLE, and BESIII and the related theoretical studies. In the charmed baryon sector, 24 singly charmed baryons and two doubly charmed baryons are listed in the current version of the review of particle physics [1]. Among them, the newest members include the $\Lambda_{c}(2860)$ [2], the five $\Omega_{c}$ states [3], and the $\Xi_{c c}^{++}$[4]. Inspired by these and other experimental discoveries, there have been extensive theoretical and lattice QCD studies on their nature and their decay and production mechanisms (see, e.g., Refs. [5-12] and references cited therein).

The magnetic moment of a baryon plays an extremely important role in understanding its internal structure. Historically, the experimental measurements of the magnetic moments of the proton and the neutron revealed that they are not point-like particles. The subsequent studies helped the establishment of the quark model as well as the theory of the strong interaction, quantum chromodynamics. Unlike those of the ground-state baryons, the magnetic

*lisheng.geng@buaa.edu.cn

Published by the American Physical Society under the terms of the Creative Commons Attribution 4.0 International license. Further distribution of this work must maintain attribution to the author(s) and the published article's title, journal citation, and DOI. Funded by SCOAP ${ }^{3}$. moments of the spin-1/2 singly charmed baryons have not been measured experimentally. Nevertheless, they have been studied in a variety of phenomenological models [13-21], QCD sum rules [22,23], the heavy baryon chiral perturbation theory (HB ChPT) [24,25], and lattice QCD simulations [26-29]. In Ref. [24], the low energy constants (LECs) are determined by the quark model and the heavy quark spin flavor symmetry and by fitting to the lattice QCD data extrapolated to the physical point. ${ }^{1}$ In this work, we will study the magnetic moments of the spin- $1 / 2$ singly charmed baryons up to the next-to-leading order (NLO) in covariant baryon chiral perturbation theory (BChPT) with the extended-on-mass shell (EOMS) renormalization scheme. The unknown LECs $g_{1,2,3,4}$ will be determined by the quark model and the heavy quark spin flavor symmetry, and the LECs $d_{2}, d_{3}$, and $d_{5}$ and $d_{6}$ by directly fitting to the lattice QCD data at unphysical pion masses [26-29]. We note that many previous studies, such as Refs. [30,31], have shown that the EOMS BChPT can provide a better description of the lattice QCD quark-mass dependent data than its noncovariant counterpart (at the same chiral order). It will be interesting to check whether the same happens in the case of the magnetic moments of singly charmed baryons.

Chiral perturbation theory (ChPT) [32], as a low-energy effective field theory of QCD, is an appropriate framework to study the magnetic moments of hadrons, particularly,

\footnotetext{
${ }^{1}$ The extrapolations in $m_{\pi}$ were performed by phenomenological fits either constant or linear or quadratic in $m_{\pi}^{2}$.
} 
their light quark mass dependence. It provides a systematic expansion of physical observables in powers of $\left(p / \Lambda_{\chi}\right)^{n_{\chi}}$, where $p$ is a generic small quantity, either meson four momenta or baryon three momenta or light quark masses, $\Lambda_{\chi}$ is the chiral symmetry breaking scale, and $n_{\chi}$ is the chiral order, defined as $n_{\chi}=4 L-2 N_{M}-N_{B}+\sum_{k} k V_{k}$ for a Feynman diagram containing $L$ loops, $N_{M}\left(N_{B}\right)$ internal meson (baryon) propagators, and $V_{k}$ vertices from $k$ th order Lagrangians. However, its application to the onebaryon sector encountered a difficulty; i.e., a systematic power counting (PC) is lost due to the large nonvanishing baryon mass $m_{0}$ in the chiral limit. Over the years, three approaches were proposed to overcome this issue, i.e., the HB [33,34], the infrared (IR) [35], and the EOMS [36] schemes. Compared to the HB ChPT, the IR and the EOMS schemes remove the power-counting breaking (PCB) terms but retain a series of nominally higher order terms to keep Lorentz invariance [35,36]. A brief summary and comparison of these three approaches can be found in Ref. [37].

In the present work, our main purpose is to study the lattice QCD data on the magnetic moments of singly charmed baryons [26-29] and perform chiral extrapolations using the EOMS BChPT, while in Refs. [26-29], the chiral extrapolations were performed with a phenomenological approach. We note that in two recent works [38,39], the lattice QCD results on the magnetic moments of the $\Xi_{c c}$ baryons have been studied using the EOMS BChPT.

This work is organized as follows. In Sec. II, we provide the effective Lagrangians and calculate the relevant Feynman diagrams up to $\mathcal{O}\left(p^{3}\right)$. Results and discussions are given in Sec. III, which is followed by a short summary in Sec. IV.

\section{THEORETICAL FORMALISM}

The magnetic moments of singly charmed baryons are defined via the matrix elements of the electromagnetic current $J_{\mu}$ as follows:

$$
\begin{aligned}
& \left\langle\psi\left(p_{f}\right)\left|J_{\mu}\right| \psi\left(p_{i}\right)\right\rangle \\
& \quad=\bar{u}\left(p_{f}\right)\left[\gamma_{\mu} F_{1}^{B}\left(q^{2}\right)+\frac{i \sigma_{\mu \nu} q^{\nu}}{2 m_{B}} F_{2}^{B}\left(q^{2}\right)\right] u\left(p_{i}\right),
\end{aligned}
$$

where $\bar{u}\left(p_{f}\right)$ and $u\left(p_{i}\right)$ are the Dirac spinors, $m_{B}$ is the singly charmed baryon mass, and $F_{1}^{B}\left(q^{2}\right)$ and $F_{2}^{B}\left(q^{2}\right)$ denote the Dirac and Pauli form factors, respectively. The four-momentum transfer is defined as $q=p_{f}-p_{i}$. At $q^{2}=0, F_{2}^{B}(0)$ is the so-called anomalous magnetic moment, $\kappa_{B}$, and the magnetic moment is $\mu_{B}=$ $\frac{m_{N}}{m_{B}}\left(\kappa_{B}+Q_{B}\right)$, where $Q_{B}$ is the charge of the singly charmed baryon, $m_{N}=940 \mathrm{MeV}$ is the nucleon mass. Note that the magnetic moments of the singly charmed baryons $\mu_{B}$ obtained in the Lattice QCD studies of Refs. [26-29] are given in units of $\mu_{N}$. Therefore, for

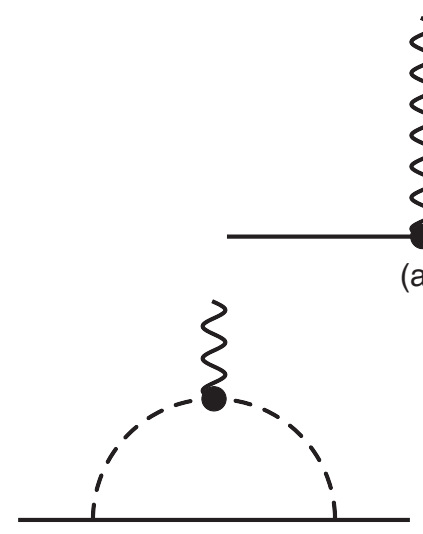

(b)

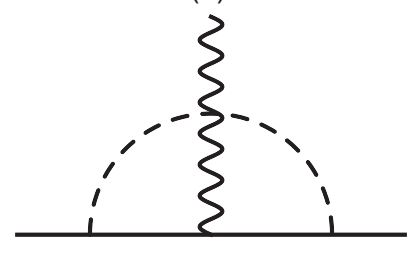

(d)

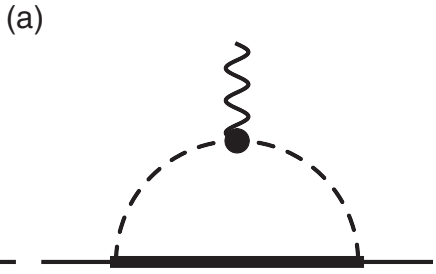

(c)

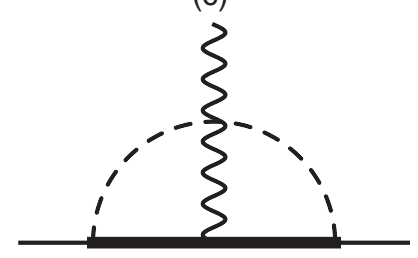

(e)
FIG. 1. Feynman diagrams contributing to the singly charmed baryon magnetic moments up to NLO. Diagram (a) contributes at LO, while the other diagrams contribute at NLO. The solid, dashed, and wiggly lines represent singly charmed baryon, Goldstone bosons, and photons, respectively. The heavy dots denote the $\mathcal{O}\left(p^{2}\right)$ vertices.

the sake of convenience, we also take $\mu_{N}$ as the units of $\mu_{B}$ when performing fits to the lattice QCD data and comparing with other predictions.

The five Feynman diagrams contributing to $\mu_{B}$ up to $\mathcal{O}\left(p^{3}\right)$ are shown in Fig. 1. The leading order contribution of $\mathcal{O}\left(p^{2}\right)$ is provided by the following Lagrangian:

$$
\begin{aligned}
& \mathcal{L}_{33}^{(2)}=\frac{d_{2}}{16 m_{\overline{3}}} \operatorname{Tr}\left(\bar{B}_{\overline{3}} \sigma^{\mu \nu} F_{\mu \nu}^{+} B_{\overline{3}}\right)+\frac{d_{3}}{16 m_{\overline{3}}} \operatorname{Tr}\left(\bar{B}_{\overline{3}} \sigma^{\mu \nu} B_{\overline{3}}\right) \operatorname{Tr}\left(F_{\mu \nu}^{+}\right), \\
& \mathcal{L}_{66}^{(2)}=\frac{d_{5}}{8 m_{6}} \operatorname{Tr}\left(\bar{B}_{6} \sigma^{\mu \nu} F_{\mu \nu}^{+} B_{6}\right)+\frac{d_{6}}{8 m_{6}} \operatorname{Tr}\left(\bar{B}_{6} \sigma^{\mu \nu} B_{6}\right) \operatorname{Tr}\left(F_{\mu \nu}^{+}\right),
\end{aligned}
$$

where the numbers in the superscript are the chiral order, $\sigma^{\mu \nu}=\frac{i}{2}\left[\gamma^{\mu}, \gamma^{\nu}\right], F_{\mu \nu}^{+}=|e|\left(u^{\dagger} Q_{h} F_{\mu \nu} u+u Q_{h} F_{\mu \nu} u^{\dagger}\right)$, $F_{\mu \nu}=\partial_{\mu} A_{\nu}-\partial_{\nu} A_{\mu}$, and $Q_{h}=\operatorname{diag}(1,0,0)$ is the charge operator of the charmed baryon, $u=\exp \left[i \Phi / 2 F_{\phi}\right]$, with the unimodular matrix containing the pseudoscalar nonet $\Phi$, and $F_{\phi}$ the pseudoscalar decay constant. In the following analysis, we take $F_{\pi}=92.4 \mathrm{MeV}, F_{K}=1.22 F_{\pi}$, and $F_{\eta}=1.3 F_{\pi} \cdot{ }^{2}$ In the $\mathrm{SU}(3)$ flavor representation, there are three kinds of singly charmed baryons, which are denoted as $B_{\overline{3}}, B_{6}$, and $B_{6}^{* \mu}$, respectively,

\footnotetext{
${ }^{2}$ The differences compared with the use of the chiral limit pseudoscalar decay constant are of higher chiral order.
} 


$$
\begin{aligned}
& B_{\overline{3}}=\left(\begin{array}{ccc}
0 & \Lambda_{c}^{+} & \Xi_{c}^{+} \\
-\Lambda_{c}^{+} & 0 & \Xi_{c}^{0} \\
-\Xi_{c}^{+} & -\Xi_{c}^{0} & 0
\end{array}\right), \quad B_{6}=\left(\begin{array}{ccc}
\Sigma_{c}^{++} & \frac{\Sigma_{c}^{+}}{\sqrt{2}} & \frac{\Xi_{c}^{\prime+}}{\sqrt{2}} \\
\frac{\Sigma_{c}^{+}}{\sqrt{2}} & \Sigma_{c}^{0} & \frac{\Xi_{c}^{\prime 0}}{\sqrt{2}} \\
\frac{\Xi_{c}^{++}}{\sqrt{2}} & \frac{\Xi_{c}^{\prime 0}}{\sqrt{2}} & \Omega_{c}^{0}
\end{array}\right), \\
& B_{6}^{* \mu}=\left(\begin{array}{ccc}
\Sigma_{c}^{*++} & \frac{\Sigma_{c}^{*+}}{\sqrt{2}} & \frac{\Xi_{c}^{*+}}{\sqrt{2}} \\
\frac{\Sigma_{c}^{*+}}{\sqrt{2}} & \Sigma_{c}^{* 0} & \frac{\Xi_{c}^{* 0}}{\sqrt{2}} \\
\frac{\Xi_{c}^{*+}}{\sqrt{2}} & \frac{\Xi_{c}^{* 0}}{\sqrt{2}} & \Omega_{c}^{* 0}
\end{array}\right) .
\end{aligned}
$$

The spin of the $B_{\overline{3}}$ and $B_{6}$ states is $1 / 2$, while the spin of the $B_{6}^{* \mu}$ states is $3 / 2$.

In the numerical analysis, we take the average of the masses for each flavor multiplet, i.e., $m_{\overline{3}}=2408 \mathrm{MeV}$, $m_{6}=2535 \mathrm{MeV}$, and $m_{6^{*}}=2602 \mathrm{MeV}$ [1]. The mass differences are $\delta_{1}=m_{6}-m_{\overline{3}}=127 \mathrm{MeV}, \delta_{2}=m_{6^{*}}-m_{\overline{3}}=$ $194 \mathrm{MeV}$, and $\delta_{3}=m_{6^{*}}-m_{6}=67 \mathrm{MeV}^{3}$

The loop diagrams arising at NLO are determined in terms of the lowest order LECs from $\mathcal{L}_{B}^{(1)}+\mathcal{L}_{M B}^{(1)}+\mathcal{L}_{M}^{(2)}$, which are,

$$
\begin{aligned}
\mathcal{L}_{B}^{(1)}= & \frac{1}{2} \operatorname{Tr}\left[\bar{B}_{\overline{3}}\left(i \not D-m_{\overline{3}}\right) B_{\overline{3}}\right]+\operatorname{Tr}\left[\bar{B}_{6}\left(i \not D-m_{6}\right) B_{6}\right]+\operatorname{Tr}\left[\bar{B}_{6}^{* \mu}\left(-g_{\mu \nu}\left(i \not D-m_{6^{*}}\right)+i\left(\gamma_{\mu} D_{\nu}+\gamma_{\nu} D_{\mu}\right)-\gamma_{\mu}\left(i \not D+m_{6^{*}}\right) \gamma_{\nu} B_{6}^{* \nu}\right],\right. \\
\mathcal{L}_{M B}^{(1)}= & \frac{g_{1}}{2} \operatorname{Tr}\left[\bar{B}_{6} \not l \gamma_{5} B_{6}\right]+\frac{g_{2}}{2} \operatorname{Tr}\left[\bar{B}_{6} \not l \gamma_{5} B_{\overline{3}}+\text { H.c. }\right]+\frac{i g_{3}}{2 F_{\phi} m_{6}^{*}} \operatorname{Tr}\left[\partial^{\alpha} \bar{B}_{6}^{* \mu} \gamma_{\alpha \mu \nu} \partial^{\nu} \Phi B_{6}+\text { H.c. }\right] \\
& +\frac{i g_{4}}{2 F_{\phi} m_{6}^{*}} \operatorname{Tr}\left[\partial^{\alpha} \bar{B}_{6}^{* \mu} \gamma_{\alpha \mu \nu} \partial^{\nu} \Phi B_{\overline{3}}+\text { H.c. }\right]+\frac{g_{5}}{2} \operatorname{Tr}\left[\bar{B}_{6}^{* \mu} \not l \gamma_{5} B_{6 \mu}^{*}\right]+\frac{g_{6}}{2} \operatorname{Tr}\left[\bar{B}_{\overline{3}} \not l \gamma_{5} B_{\overline{3}}\right] \\
\mathcal{L}_{M}^{(2)}= & \frac{F_{\phi}^{2}}{4} \operatorname{Tr}\left[\nabla_{\mu} U\left(\nabla^{\mu} U\right)^{\dagger}\right]
\end{aligned}
$$

with

$$
\begin{aligned}
D_{\mu} B & =\partial_{\mu} B+\Gamma_{\mu} B+B \Gamma_{\mu}^{T}, \\
\Gamma_{\mu} & =\frac{1}{2}\left(u^{\dagger} \partial_{\mu} u+u \partial_{\mu} u^{\dagger}\right)-\frac{i}{2}\left(u^{\dagger} v_{\mu} u+u v_{\mu} u^{\dagger}\right)=-i e Q_{h} A_{\mu}, \\
u_{\mu} & =i\left(u^{\dagger} \partial_{\mu} u-u \partial_{\mu} u^{\dagger}\right)+\left(u^{\dagger} v_{\mu} u-u v_{\nu} u^{\dagger}\right), \\
U & =u^{2}=e^{\frac{i \Phi}{F_{\phi}}}, \quad \nabla_{\mu} U=\partial_{\mu} U+i e A_{\mu}\left[Q_{l}, U\right],
\end{aligned}
$$

where $v_{\mu}$ stands for the vector source, and the charge matrix for the light $u, d, s$ quarks is $Q_{l}=\operatorname{diag}(2 / 3,-1 / 3,-1 / 3)$. The total spin of the light quarks is 0 for the singly charmed baryon in the $B_{\overline{3}}$ state. Considering parity and angular momentum conservation, the $\bar{B}_{\overline{3}} B_{\overline{3}} \Phi$ vertex is forbidden, i.e., $g_{6}=0$. Here, we would like to stress that for the Lagrangians of $\bar{B}_{6}^{*} B_{6 / 3} \Phi$, we choose to work with the socalled "consistent" couplings, introduced in Refs. [40,41]. In Ref. [42], it was shown that for the octet baryon magnetic moments, the use of the consistent couplings allowed one to obtain both a proper description of the experimental data and a proper convergence behavior. Indeed, we find that in the present work it also provides a better description of the lattice QCD magnetic moments of singly charmed baryons, but the improvement is not that dramatic as in the case of the ground state octet baryons.

For the $B_{\overline{3}}$ and $B_{6}$ states, the tree level contributions of the magnetic moments can be easily obtained from Eq. (2), which are:

\footnotetext{
${ }^{3}$ In the heavy quark limit, these mass differences vanish.
}

$$
\kappa_{\overline{3}}^{(a, 2)}=\alpha_{\overline{3}} d_{2}+\beta_{\overline{3}} d_{3}, \quad \kappa_{6}^{(a, 2)}=\alpha_{6} d_{5}+\beta_{6} d_{6} .
$$

The values of $\alpha_{\overline{3}}, \beta_{\overline{3}}, \alpha_{6}$, and $\beta_{6}$ are tabulated in Tables I and II. The four LECs $d_{2}, d_{3}, d_{5}$, and $d_{6}$ will be determined by fitting to the lattice QCD data of Refs. [26-29].

TABLE I. Coefficients of the tree level contributions of Eq. (6) for the $B_{\overline{3}}$ states.

\begin{tabular}{cccc}
\hline \hline & $\Lambda_{c}^{+}$ & $\Xi_{c}^{+}$ & $\Xi_{c}^{0}$ \\
\hline$\alpha_{\overline{3}}$ & $\frac{1}{2}$ & $\frac{1}{2}$ & 0 \\
$\beta_{\overline{3}}$ & 1 & 1 & 1 \\
\hline \hline
\end{tabular}

TABLE II. Coefficients of the tree level contributions of Eq. (6) for the $B_{6}$ states.

\begin{tabular}{ccccccc}
\hline \hline & $\Sigma_{c}^{++}$ & $\Sigma_{c}^{+}$ & $\Sigma_{c}^{0}$ & $\Xi_{c}^{\prime+}$ & $\Xi_{c}^{\prime 0}$ & $\Omega_{c}^{0}$ \\
\hline$\alpha_{6}$ & 1 & $\frac{1}{2}$ & 0 & $\frac{1}{2}$ & 0 & 0 \\
$\beta_{6}$ & 1 & 1 & 1 & 1 & 1 & 1 \\
\hline \hline
\end{tabular}


At $\mathcal{O}\left(p^{3}\right)$, the loop contributions to the magnetic moments, which come from diagrams (b), (c), (d), and (e) of Fig. 1, are written as,

$$
\begin{aligned}
\kappa_{\overline{3}}^{(3)}= & \frac{1}{4}\left(\sum_{\phi=\pi, K} \frac{g_{2}^{2}}{F_{\phi}^{2}} \xi_{B_{\overline{3}} \phi, \delta_{1}}^{(3, b)} H_{B_{\overline{3}}}^{(b)}\left(\delta_{1}, m_{\phi}\right)+\sum_{\phi=\pi, K} \frac{g_{4}^{2}}{F_{\phi}^{2}} \xi_{B_{\overline{3}} \phi, \delta_{2}}^{(3, c)} H_{B_{\overline{3}}}^{(c)}\left(\delta_{2}, m_{\phi}\right)\right. \\
& \left.+\sum_{\phi=\pi, K, \eta} \frac{g_{2}^{2}}{F_{\phi}^{2}} \xi_{B_{\overline{3}} \phi, \delta_{1}}^{(3, d)} H_{B_{\overline{3}}}^{(d)}\left(\delta_{1}, m_{\phi}\right)+\sum_{\phi=\pi, K, \eta} \frac{g_{4}^{2}}{F_{\phi}^{2}} \xi_{B_{\overline{3}} \phi, \delta_{2}}^{(3, e)} H_{B_{\overline{3}}}^{(e)}\left(\delta_{2}, m_{\phi}\right)\right), \\
\kappa_{6}^{(3)}= & \frac{1}{4}\left(\sum_{\phi=\pi, K} \frac{g_{1}^{2}}{F_{\phi}^{2}} \xi_{B_{6} \phi}^{(3, b)} H_{B_{6}}^{(b)}\left(0, m_{\phi}\right)+\sum_{\phi=\pi, K} \frac{g_{2}^{2}}{F_{\phi}^{2}} \xi_{B_{6} \phi, \delta_{1}}^{(3, b)} H_{B_{6}}^{(b)}\left(\delta_{1}, m_{\phi}\right)\right. \\
& +\sum_{\phi=\pi, K} \frac{g_{3}^{2}}{F_{\phi}^{2}} \xi_{B_{6} \phi, \delta_{3}}^{(3, c)} H_{B_{6}}^{(c)}\left(\delta_{3}, m_{\phi}\right)+\sum_{\phi=\pi, K, \eta} \frac{g_{1}^{2}}{F_{\phi}^{2}} \xi_{B_{6} \phi}^{(3, d)} H_{B_{6}}^{(d)}\left(0, m_{\phi}\right) \\
& \left.+\sum_{\phi=\pi, K, \eta} \frac{g_{2}^{2}}{F_{\phi}^{2}} \xi_{B_{6} \phi, \delta_{1}}^{(3, d)} H_{B_{6}}^{(d)}\left(\delta_{1}, m_{\phi}\right)+\sum_{\phi=\pi, K, \eta} \frac{g_{3}^{2}}{F_{\phi}^{2}} \xi_{B_{6} \phi, \delta_{3}}^{(3, e)} H_{B_{6}}^{(e)}\left(\delta_{3}, m_{\phi}\right)\right),
\end{aligned}
$$

with the coefficients $\xi_{B_{\overline{3}} \phi, \delta_{i}}^{(3 ; b, c)}$ and $\xi_{B_{6} \phi, \delta_{i}}^{(3 ; b, d, e)}$ listed in Tables III and IV. The explicit expressions of the loop functions $H_{B_{\overline{3}}}^{(b, c, d, e)}\left(\delta_{i}, m_{\phi}\right)$ and $H_{B_{6}}^{(b, c, d, e)}\left(\delta_{i}, m_{\phi}\right)$ can be found in the Appendix.

Once we obtain the loop functions in the EOMS scheme, we can easily obtain their HB counterparts by performing $1 / m_{0}$ expansions. We have checked that our results agree with those of Ref. [24]. In the following section, for the sake of comparison, we study also the performance of the HB ChPT in describing the lattice QCD data of Refs. [26-29]. It should be noted that in the following section, unless otherwise stated, the HB ChPT results refer to the ones obtained in the present work, not those of Ref. [24]. In the present work, due to the limited lattice QCD data [26-29], we will treat the differences between

TABLE III. Coefficients of the loop contributions of Eq. (7) for the $B_{\overline{3}}$ states.

\begin{tabular}{lccc}
\hline \hline & $\Lambda_{c}^{+}$ & $\Xi_{c}^{+}$ & $\Xi_{c}^{0}$ \\
\hline$\xi_{B_{3} \pi, \delta_{1}}^{(3, b)}$ & 0 & 1 & -1 \\
$\xi_{B_{3} K, \delta_{1}}^{(3, b)}$ & 1 & 0 & -1 \\
$\xi_{B_{3} \pi, \delta_{2}}^{(3, c)}$ & 0 & 1 & -1 \\
$\xi_{B_{3} K, \delta_{2}}^{(3,, c)}$ & 1 & 0 & -1 \\
$\xi_{B_{3} \pi, \delta_{1}}^{(3, d)}$ & 6 & $\frac{1}{2}$ & 1 \\
$\xi_{B_{3} K, \delta_{1}}^{(3, d)}$ & 1 & 5 & 1 \\
$\xi_{B_{3} \eta, \delta_{1}}^{(3, d)}$ & 0 & $\frac{3}{2}$ & 0 \\
$\xi_{B_{3} \pi, \delta_{2}}^{(3, e)}$ & 6 & $\frac{1}{2}$ & 1 \\
$\xi_{B_{3} K, \delta_{2}}^{(3, e)}$ & 1 & 5 & 1 \\
$\xi_{B_{3} \eta, \delta_{2}}^{(3, e)}$ & 0 & $\frac{3}{2}$ & 0 \\
\hline \hline
\end{tabular}

the results of the HB ChPT and those of the EOMS BChPT as our estimates of inherent systematic uncertainties originated from chiral truncations.

\section{RESULTS AND DISCUSSIONS}

In this section, we determine the LECs $d_{2}, d_{3}, d_{5}$, and $d_{6}$ by fitting to the lattice QCD data of Refs. [26-29], which are collected in Table $\mathrm{V}$ for the sake of easy reference. Because of the limited lattice QCD data, the

\begin{tabular}{|c|c|c|c|c|c|c|}
\hline & $\Sigma_{c}^{++}$ & $\Sigma_{c}^{+}$ & $\Sigma_{c}^{0}$ & $\Xi_{c}^{\prime+}$ & $\Xi_{c}^{\prime 0}$ & $\Omega_{c}^{0}$ \\
\hline$\overline{\xi_{B, \pi}^{(3, b)}}$ & 1 & 0 & -1 & $\frac{1}{2}$ & $-\frac{1}{2}$ & 0 \\
\hline$\xi_{B_{6} K}^{(3, b)}$ & 1 & $\frac{1}{2}$ & 0 & 0 & $-\frac{1}{2}$ & -1 \\
\hline$\xi_{B_{6} \pi, \delta_{1}}^{(3, b)}$ & 2 & 0 & -2 & 1 & -1 & 0 \\
\hline$\xi_{B_{6} K, \delta_{1}}^{(3, b)}$ & 2 & 1 & 0 & 0 & -1 & -2 \\
\hline$\xi_{B_{6} \pi, \delta_{3}}^{(3, c)}$ & 1 & 0 & -1 & $\frac{1}{2}$ & $-\frac{1}{2}$ & 0 \\
\hline$\xi_{B_{6} K, \delta_{3}}^{(3, c)}$ & 1 & $\frac{1}{2}$ & 0 & 0 & $-\frac{1}{2}$ & -1 \\
\hline$\xi_{B_{6} \pi}^{(3, d)}$ & 3 & 2 & 1 & $\frac{1}{4}$ & $\frac{1}{2}$ & 0 \\
\hline$\xi_{B_{6} K}^{(3, d)}$ & 1 & $\frac{1}{2}$ & 0 & $\frac{5}{2}$ & $\frac{1}{2}$ & 1 \\
\hline$\xi_{B_{6} \eta}^{(3, d)}$ & $\frac{2}{3}$ & $\frac{1}{3}$ & 0 & $\frac{1}{12}$ & 0 & 0 \\
\hline$\xi_{B_{6} \pi, \delta_{1}}^{(3, d)}$ & 2 & 2 & 2 & $\frac{1}{2}$ & 1 & 0 \\
\hline$\xi_{B_{6} K, \delta_{1}}^{(3, d)}$ & 2 & 1 & 0 & 1 & 1 & 2 \\
\hline$\xi_{B_{6} \eta, \delta_{1}}^{(3, d)}$ & 0 & 0 & 0 & $\frac{3}{2}$ & 0 & 0 \\
\hline$\xi_{B_{6} \pi, \delta_{3}}^{(3, e)}$ & 3 & 2 & 1 & $\frac{1}{4}$ & $\frac{1}{2}$ & 0 \\
\hline$\xi_{B_{6} K, \delta_{3}}^{(3, e)}$ & 1 & $\frac{1}{2}$ & 0 & $\frac{5}{2}$ & $\frac{1}{2}$ & 1 \\
\hline${\underline{B_{6} \eta, \delta_{3}}}^{(3 .}$ & $\frac{2}{3}$ & $\frac{1}{3}$ & 0 & $\frac{1}{12}$ & 0 & 0 \\
\hline
\end{tabular}
other LECs $g_{1-4}$ are fixed by the quark model and the heavy quark spin flavor symmetry. Their values are

TABLE IV. Coefficients of the loop contributions of Eq. (7) for the $B_{6}$ states. 
TABLE V. Magnetic moments of singly charmed baryons at different $m_{\pi}$ [26-29], in units of nuclear magneton, $\mu_{N}$.

\begin{tabular}{lccccccc}
\hline \hline$m_{\pi}(\mathrm{MeV})$ & $\Xi_{c}^{+}$ & $\Xi_{c}^{0}$ & $\Sigma_{c}^{++}$ & $\Sigma_{c}^{0}$ & $\Xi_{c}^{\prime+}$ & $\Xi_{c}^{\prime 0}$ & $\Omega_{c}^{0}$ \\
\hline Phys. & $\ldots$ & $\ldots$ & $1.499(202)$ & $-0.875(103)$ & $\ldots$ & $\ldots$ & $-0.667(96)$ \\
156 & $0.235(25)$ & $0.192(17)$ & $\ldots$ & $\ldots$ & $0.315(141)$ & $-0.599(71)$ & $-0.688(31)$ \\
300 & $\ldots$ & $\ldots$ & $1.867(388)$ & $-0.929(206)$ & $\ldots$ & $\ldots$ & $-0.640(55)$ \\
410 & $\ldots$ & $\ldots$ & $1.591(358)$ & $-0.897(223)$ & $\ldots$ & $\ldots$ & $-0.621(44)$ \\
570 & $\ldots$ & $\ldots$ & $1.289(161)$ & $-0.724(80)$ & $\ldots$ & $\ldots$ & $-0.658(46)$ \\
700 & $\ldots$ & $\ldots$ & $1.447(125)$ & $-0.757(67)$ & $\ldots$ & $\ldots$ & $-0.701(56)$ \\
\hline \hline
\end{tabular}

TABLE VI. LECs $d_{2}, d_{3}, d_{5}$, and $d_{6}$ determined by fitting to the lattice QCD data, with $g_{1-4}$ fixed. The numbers in the brackets are the statistical uncertainties at the $68 \%$ confidence level originated from the uncertainties of the lattice QCD data. In case 1 all the allowed intermediate baryons in the loop diagrams are taken into account, while in case 2 only intermediate baryons of the same type as those of the external baryons in the loop diagrams are considered.

\begin{tabular}{lcclll}
\hline \hline & \multicolumn{2}{c}{ Case 1 } & & \multicolumn{2}{c}{ Case 2 } \\
\cline { 2 - 3 } \cline { 5 - 6 } & EOMS 1 & HB 1 & & EOMS 2 & HB 2 \\
\hline$d_{2}$ & $-1.49(15)$ & $-2.32(15)$ & & $-1.78(15)$ & $-1.78(15)$ \\
$d_{3}$ & $0.63(4)$ & $0.65(4)$ & & $0.49(4)$ & $0.49(4)$ \\
$d_{5}$ & $8.28(34)$ & $13.49(34)$ & & $5.38(34)$ & $8.69(34)$ \\
$d_{6}$ & $-4.17(5)$ & $-4.93(5)$ & & $-2.83(5)$ & $-3.40(5)$ \\
$g_{1}$ & 0.98 & 0.98 & & 0.98 & 0.98 \\
$g_{2}$ & -0.60 & -0.60 & & 0 & 0 \\
$g_{3}$ & 0.85 & 0.85 & & 0.85 & 0.85 \\
$g_{4}$ & 1.04 & 1.04 & & 0 & 0 \\
$\chi_{\min }^{2} /$ d.o.f. & 5.63 & 14.56 & & 1.93 & 3.82 \\
\hline \hline
\end{tabular}

$g_{1}=0.98, g_{2}=-\sqrt{\frac{3}{8}} g_{1}=-0.60, g_{3}=\frac{\sqrt{3}}{2} g_{1}=0.85$, and $g_{4}=-\sqrt{3} g_{2}=1.04[43,44] .^{4}$ In our least-squares fit, the $\chi^{2}$ as a function of the LECs is defined as

$$
\chi^{2}\left(C_{X}\right)=\sum_{i=1}^{n} \frac{\left(\mu_{i}^{\mathrm{th}}\left(C_{X}\right)-\mu_{i}^{\mathrm{LQCD}}\right)^{2}}{\sigma_{i}^{2}}
$$

where $C_{X}$ denote all the LECs, $\sigma_{i}$ correspond to the uncertainty of each lattice QCD datum, $\mu_{i}^{\text {th }}\left(C_{X}\right)$ and $\mu_{i}^{\mathrm{LQCD}}$ stand for the magnetic moments obtained in the $\mathrm{BChPT}$ and those of the lattice QCD in Table V, respectively.

In order to decompose the contributions of loop diagrams, we will consider two cases. In case 1 , all the allowed intermediate baryons are taken into account, while in case 2 , only intermediate baryons of the same type as

\footnotetext{
${ }^{4}$ If we used the lattice QCD determination of $g_{4}=0.71 \pm 0.13$ $[45,46]$ and the heavy quark symmetry to obtain $g_{1}, g_{2}$, and $g_{3}$, we would have obtained smaller loop corrections and thus a better convergence pattern, but all our conclusions would remain essentially unchanged.
}

those of the external baryons are considered, namely, there are no loop contributions to the magnetic moments of the triplet baryons, while in calculating the loop contributions to the magnetic moments of the sextet baryons, only intermediate sextet baryons are considered. Fitting to the lattice QCD data of Table V and with $g_{1-4}$ fixed, the resulting LECs and $\chi^{2} /$ d.o.f. are listed in Table VI. The predicted magnetic moments as a function of $m_{\pi}^{2}$ are plotted in Figs. 2 and 3 in comparison with the lattice QCD data for the antitriplet and sextet baryons, respectively. At first sight, the EOMS BChPT descriptions of the lattice QCD data seem to be better than those of the HB ChPT in both cases, consistent with previous studies [30,31]. Nevertheless, because of the limited lattice QCD data and the relatively large number of unknown LECs, the statistical significance of such an improvement is not very significant. As a result, the differences between both results may better be viewed as inherent systematic uncertainties originated from chiral truncations. In addition, we note that the contributions of the diagrams where the photon couples to intermediate baryons, which appear only at NNLO in HB ChPT, are indeed relatively small. ${ }^{5}$

As shown in Table VI and Figs. 2 and 3, in general the description of the lattice QCD data is better in case 2 than in case 1 . Furthermore, the convergence pattern in case 2 is also better than that in case $1,{ }^{6}$ with probably the exception of $\Sigma_{c}^{0}$, as has been noted in Ref. [24] as well. Therefore, we decide to take the predictions of case 2 as our final results. ${ }^{7}$

In Figs. 4 and 5, we compare the predicted magnetic moments of all the singly charmed baryons at the physical point with those obtained in other approaches. The shaded light-blue bands indicate the statistical uncertainties originated from the fitted LECs with their $1 \sigma$ confidence intervals. For the sake of comparison, we also give the

\footnotetext{
${ }^{5}$ Note that we do not fit to the lattice QCD data obtained at $m_{\pi}=700 \mathrm{MeV}$, which are probably out of the range of validity of NLO ChPT. Furthermore, as can be seen in Fig. 3, the difference between the lattice QCD value and the ChPT prediction for $\mu_{\Xi_{c}^{\prime \prime}}$ is somehow relatively large. Thus, we do not include the lattice QCD magnetic moment of $\Xi_{c}^{\prime 0}$ in our fitting as well.

${ }^{6}$ The convergence will be even better if the $g_{2}$ and $g_{4}$ couplings are smaller than we used as indicated by the lattice QCD data.

${ }^{7}$ Again, the differences between case 1 and case 2 may be taken as systematic uncertainties.
} 

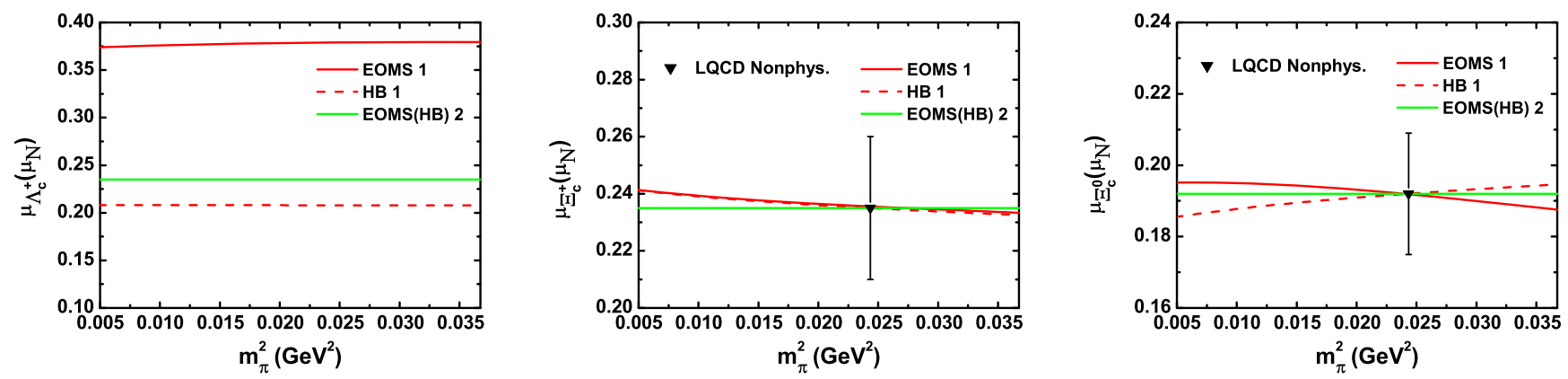

FIG. 2. Magnetic moments of the singly charmed antitriplet baryons as a function of $m_{\pi}^{2}$. The solid black nablas represent the corresponding lattice QCD data that are fitted.
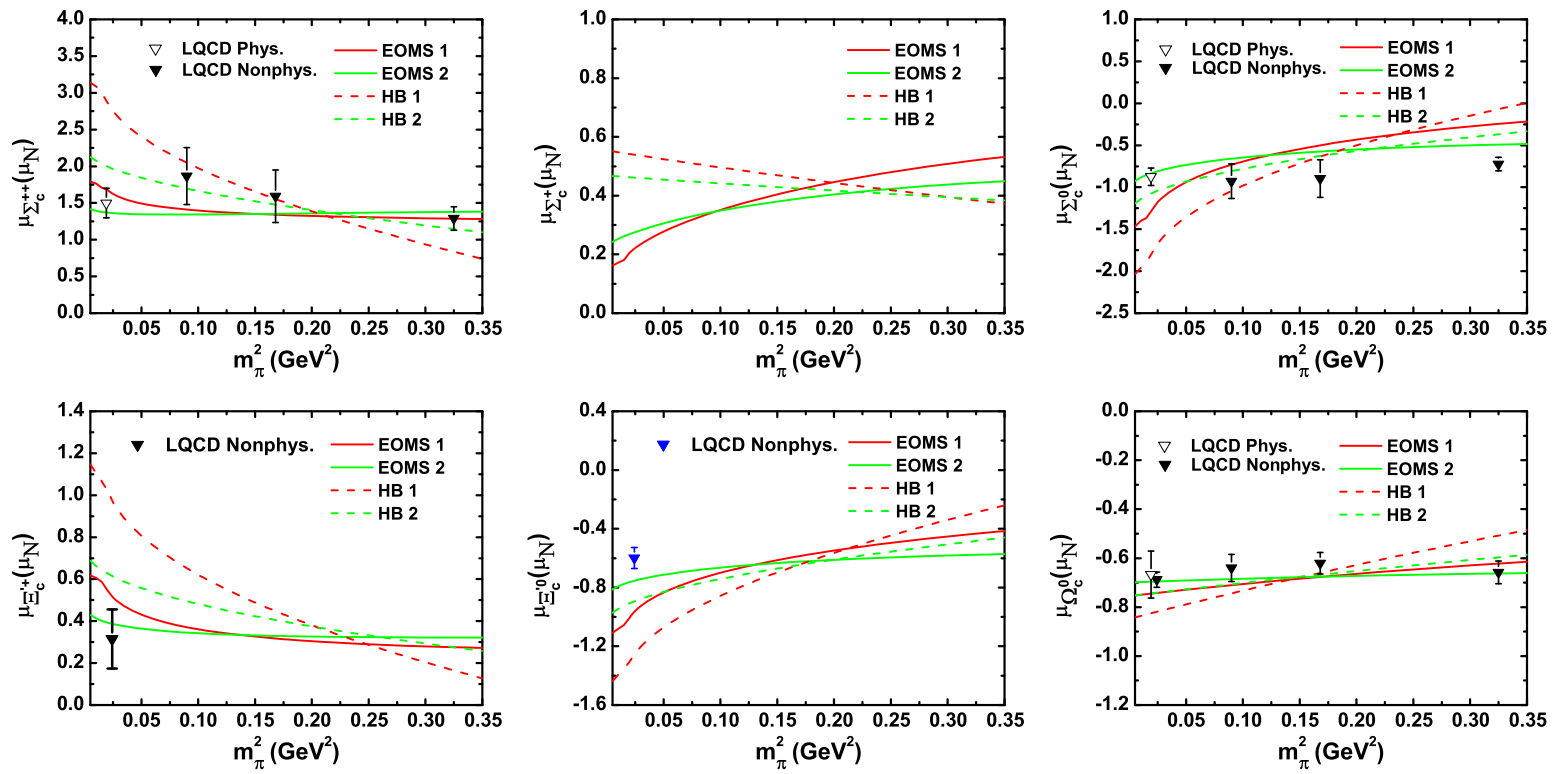

FIG. 3. Magnetic moments of the singly charmed sextet baryons as a function of $m_{\pi}^{2}$. The solid black nablas refer to the corresponding lattice QCD data fitted. The hollow nablas stand for the lattice QCD physical values. The blue nablas denote the lattice QCD data not used in our fitting.

results obtained in the present work using the HB ChPT. Note that our present study is at $\mathcal{O}\left(p^{3}\right)$, while that of Ref. [24] is at $\mathcal{O}\left(p^{4}\right)$. In addition, we have chosen different strategies to determine some of the LECs. In Ref. [24], the lattice QCD extrapolated data were used to determine $d_{2}$, $d_{3}, d_{5}$, and $d_{6}$, while in our work we have used the lattice QCD data at unphysical baryon masses. We note that the results of different approaches are rather scattered. Clearly, further experimental or lattice QCD studies are needed to discriminate between different theoretical approaches.

For both the antitriplet and sextet baryons, our predictions are in most cases smaller in absolute values than those obtained by other approaches. In addition, because case 2 is in fact a tree level study, therefore, our HB and EOMS results are the same. However, our results for the sextet baryons are in better agreement with those of the HB ChPT of Ref. [24], despite the differences pointed out above. On the other hand, there are some sizable differences between the results obtained in the present work in the EOMS scheme and those in the HB scheme, reflecting the fact that relativistic corrections are sizable, which could be taken as systematic uncertainties.

We should mention that for the results of Lattice QCD appearing in Fig. 4 and Fig. 5 we only used their physics values obtained from linear extrapolations, although they performed fits both linear and quadratic in $m_{\pi}^{2}$.

\section{HEAVY QUARK SPIN FLAVOR SYMMETRY AND ITS BREAKING}

In this section, we discuss the heavy quark spin flavor symmetry and its breaking and their impact on the magnetic moments of the antitriplet and sextet baryons at $\mathcal{O}\left(p^{3}\right)$. In Tables VII and VIII, we decompose the loop contributions into those from the intermediate $\overline{3}, 6$, and $6^{*}$ states, respectively. We note that the HB ChPT contributions to 

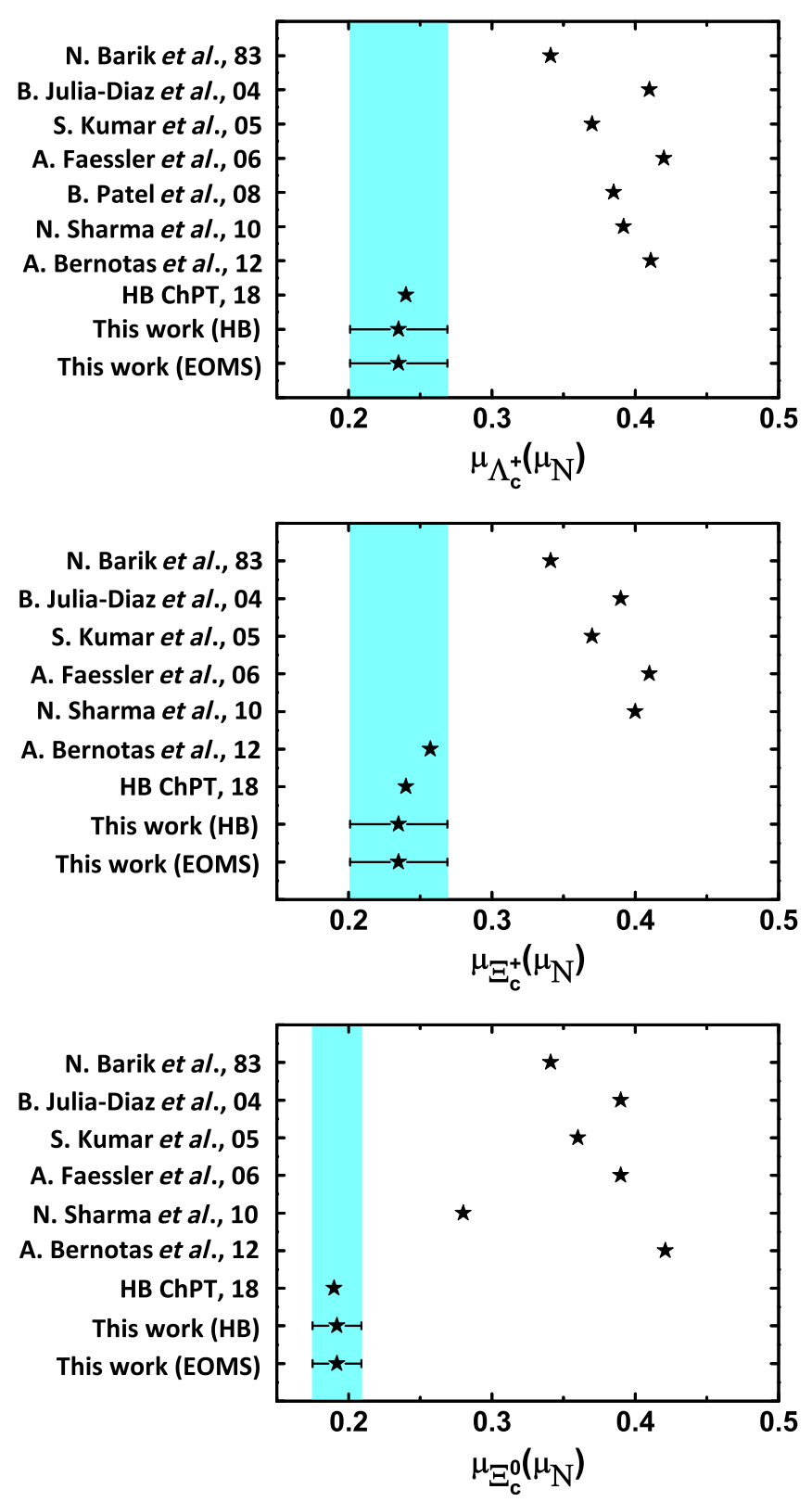

FIG. 4. Magnetic moments of the antitriplet baryons obtained in different approaches. The light-blue bands denote the uncertainties of the EOMS BChPT originated from the fitted LECs within their $1 \sigma$ confidence intervals. The others are taken from the independent-quark model [13] (N. Barik et al., 83), the relativistic quark model [14] (B. Julia-Diaz et al., 04), the effective mass and screened charge scheme [15] (S. Kumar et al., 05), the relativistic three-quark model [16] (A. Faessler et al., 06), the hyper central model [17] (B. Patel et al., 08), the chiral constituent quark model [18] (N. Sharma et al., 10), the bag model [19] (A. Bernotas et al., 12), and the HB ChPT [24] (HB ChPT, 18).

the triplet baryons from the intermediate 6 and $6^{*}$ baryons almost cancel each other at $\mathcal{O}\left(p^{3}\right)$. Thus, at this order, the net loop corrections are quite small. As a matter of fact, the nice cancellation exhibited by the HB ChPT is a direct consequence of heavy quark spin symmetry. In the

heavy quark limit, i.e., $\delta_{1}=\delta_{2}=0$, the cancellation will be exact.

On the other hand, in the EOMS scheme, because of the existence of higher order (compared to HB ChPT) relativistic corrections, heavy quark spin flavor symmetry is explicitly broken by terms of $1 / m_{\overline{3}}^{n}$ with $n \geq 1$. For the diagrams with a photon coupling to an intermediate baryon, the contributions start at $1 / m_{\overline{3}}$. As a result, these diagrams give vanishing contributions in the HB scheme, while in the EOMS scheme, they are finite (though relatively small) as shown below ${ }^{8}$ :

$$
\begin{aligned}
g_{2}^{2} & \cdot H_{B_{\overline{3}}}^{(d)}\left(0, m_{\phi}\right) \\
& =g_{2}^{2} m_{\overline{3}} \cdot\left(\frac{\log \left(\frac{m_{\overline{3}}^{2}}{m_{\phi}^{2}}\right)-2}{4 \pi^{2} m_{\overline{3}}}+\mathcal{O}\left(\frac{1}{m_{\overline{3}}^{2}}\right)\right), \\
g_{4}^{2} & \cdot H_{B_{\overline{3}}}^{(e)}\left(0, m_{\phi}\right) \\
& =3 g_{2}^{2} \cdot H_{B_{\overline{3}}}^{(c)}\left(0, m_{\phi}\right) \\
& =g_{2}^{2} m_{\overline{3}} \cdot\left(\frac{48 \log \left(\frac{m_{\overline{3}}^{2}}{m_{\phi}^{2}}\right)+156 \log \left(\frac{\mu^{2}}{m_{\overline{3}}^{2}}\right)-1}{864 \pi^{2} m_{\overline{3}}}+\mathcal{O}\left(\frac{1}{m_{\overline{3}}^{2}}\right)\right) .
\end{aligned}
$$

For the diagrams with a photon coupling to an intermediate meson, the contributions have the following form:

$$
\begin{aligned}
& g_{2}^{2} \cdot H_{B_{\overline{3}}}^{(b)}\left(0, m_{\phi}\right) \\
& \quad=g_{2}^{2} m_{\overline{3}} \cdot\left(-\frac{m_{\phi}}{4 \pi}+\frac{2 \log \left(\frac{m_{\overline{3}}^{2}}{m_{\phi}^{2}}\right)-1}{4 \pi^{2} m_{\overline{3}}} m_{\phi}^{2}+\mathcal{O}\left(\frac{1}{m_{\overline{3}}^{2}}\right)\right), \\
& g_{4}^{2} \cdot H_{B_{\overline{3}}}^{(c)}\left(0, m_{\phi}\right) \\
& =3 g_{2}^{2} \cdot H_{B_{\overline{3}}}^{(c)}\left(0, m_{\phi}\right) \\
& =g_{2}^{2} m_{\overline{3}} \cdot\left(\frac{m_{\phi}}{4 \pi}-\frac{9 \log \left(\frac{m_{\overline{3}}^{2}}{m_{\phi}^{2}}\right)+33 \log \left(\frac{\mu^{2}}{m_{\overline{3}}^{2}}\right)+59}{432 \pi^{2} m_{\overline{3}}} m_{\phi}^{2}+\mathcal{O}\left(\frac{1}{m_{\overline{3}}^{2}}\right)\right) .
\end{aligned}
$$

It is clear that at the order of $1 / m_{\overline{3}}^{0}$, the contributions from the 6 state and $6^{*}$ state are the same but of opposite signs, while at $1 / m_{\overline{3}}$, they are different and break the nice cancellation exhibited in the HB scheme.

\section{SUMMARY}

Motivated by the recent experimental progress on heavy flavor hadrons, we have studied the magnetic moments of the singly charmed baryons in the covariant baryon chiral perturbation theory (BChPT) with the extended-on-mass-

\footnotetext{
${ }^{8}$ Note that the $m_{\overline{3}}$ in the above equations outside the brackets is from the baryon field normalization.
} 

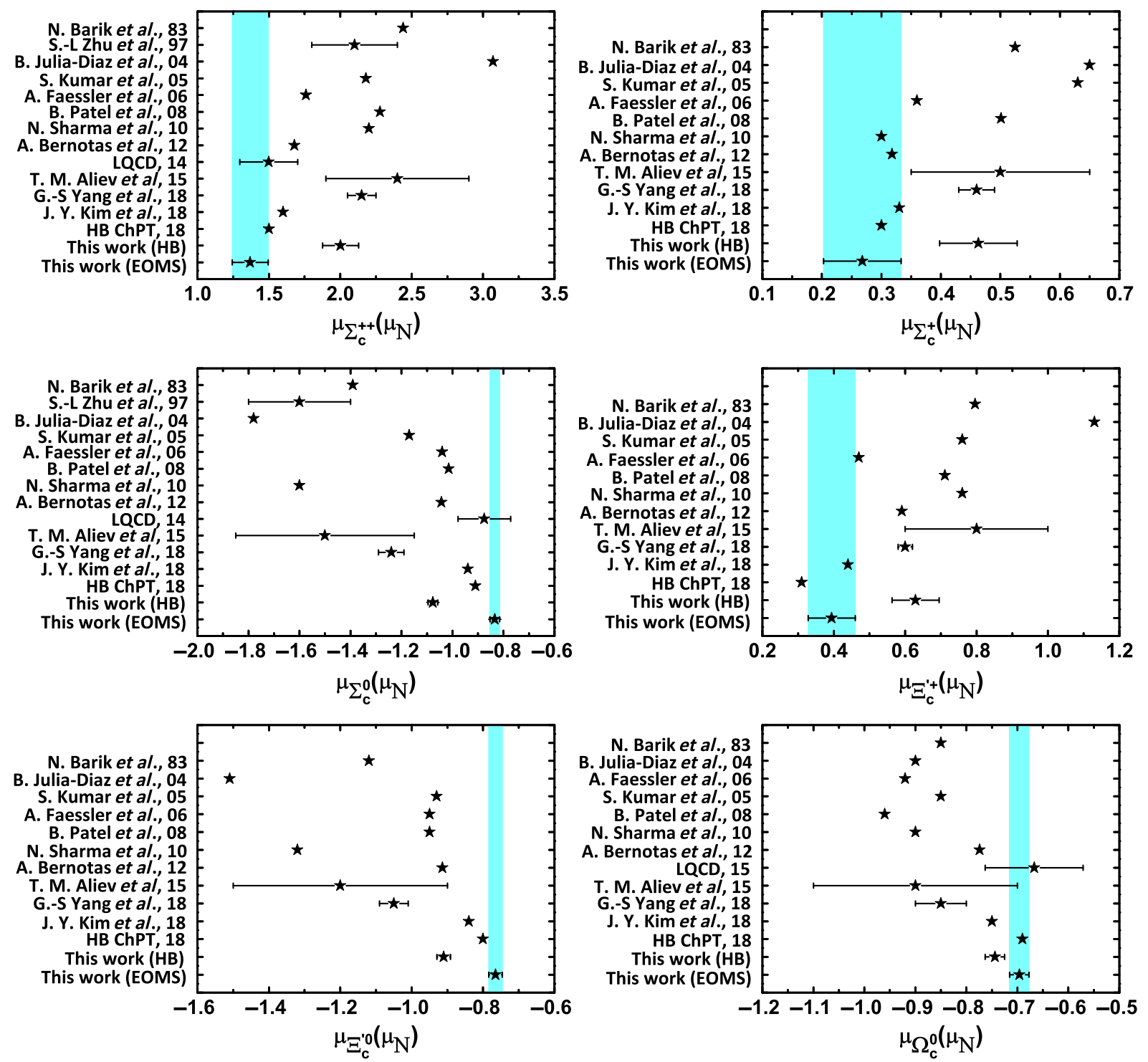

FIG. 5. Same as Fig. 4, but for the sextet baryons. Additional data are taken from the QCD sum rules [22] (S.-L. Zhu et al., 97), the light cone QCD sum rules [23] (T. M. Aliev et al., 15), the mean-field approach [20] (G.-S. Yang et al., 18), the self-consistent SU(3) chiral quark-soliton model [21] (J. Y. Kim et al., 18), lattice QCD simulations [26] (LQCD, 14), and [29] (LQCD, 15).

TABLE VII. Decomposition of the loop contributions to the magnetic moments of singly charmed baryons calculated with the central values of the LECs given in Table VI. The subscript $\overline{3}, 6$, and $6^{*}$ denote the loop diagrams with the intermediate $\overline{3}$, 6 , and $6^{*}$ states at $\mathcal{O}\left(p^{3}\right)$, respectively.

\begin{tabular}{|c|c|c|c|c|c|c|c|c|c|c|c|c|}
\hline & & \multicolumn{5}{|c|}{ EOMS 1} & \multicolumn{5}{|c|}{ HB 1} & \multirow[b]{2}{*}{ LQCD $[26,29]$} \\
\hline & & $\mathcal{O}\left(p^{2}\right)$ & $\mathcal{O}\left(p^{3}\right)_{\overline{3}}$ & $\mathcal{O}\left(p^{3}\right)_{6}$ & $\mathcal{O}\left(p^{3}\right)_{6^{*}}$ & $\mu_{\mathrm{tot}}$ & $\mathcal{O}\left(p^{2}\right)$ & $\mathcal{O}\left(p^{3}\right)_{\overline{3}}$ & $\mathcal{O}\left(p^{3}\right)_{6}$ & $\mathcal{O}\left(p^{3}\right)_{6^{*}}$ & $\mu_{\mathrm{tot}}$ & \\
\hline \multirow[t]{3}{*}{$B_{\overline{3}}$} & $\mu_{\Lambda_{c}^{+}}$ & 0.35 & $\ldots$ & -0.05 & 0.08 & 0.38 & 0.19 & $\ldots$ & -0.26 & 0.28 & 0.21 & $\ldots$ \\
\hline & $\mu_{\Xi_{c}^{+}}$ & 0.35 & $\ldots$ & 0.12 & -0.23 & 0.24 & 0.19 & $\ldots$ & -0.17 & 0.22 & 0.24 & $\ldots$ \\
\hline & $\mu_{\Xi_{c}^{0}}$ & 0.25 & $\cdots$ & 0.16 & -0.22 & 0.19 & 0.25 & $\cdots$ & 0.43 & -0.50 & 0.19 & $\cdots$ \\
\hline \multirow[t]{6}{*}{$B_{6}$} & $\mu_{\Sigma_{c}^{++}}$ & 2.27 & -0.25 & -0.44 & 0.13 & 1.71 & 3.92 & -0.32 & -0.99 & 0.29 & 2.90 & $1.499(202)$ \\
\hline & $\mu_{\Sigma_{c}^{+}}$ & 0.36 & -0.11 & -0.09 & 0.04 & 0.20 & 1.04 & -0.24 & -0.35 & 0.09 & 0.54 & $\ldots$ \\
\hline & $\mu_{\Sigma_{c}^{0}}$ & -1.55 & 0.04 & 0.26 & -0.04 & -1.29 & -1.83 & -0.17 & 0.29 & -0.11 & -1.82 & $-0.875(103)$ \\
\hline & $\mu_{\Xi_{c}^{\prime+}}^{s^{+}}$ & 0.36 & 0.12 & 0.11 & -0.04 & 0.55 & 1.04 & 0.08 & -0.15 & 0.05 & 1.02 & $\ldots$ \\
\hline & $\mu_{\Xi_{c}^{\prime 0}}$ & -1.55 & 0.24 & 0.38 & -0.10 & -1.03 & -1.83 & 0.16 & 0.49 & -0.14 & -1.32 & $\ldots$ \\
\hline & $\mu_{\Omega_{c}^{0}}$ & -1.55 & 0.45 & 0.51 & -0.15 & -0.74 & -1.83 & 0.49 & 0.70 & -0.18 & -0.82 & $-0.667(96)$ \\
\hline
\end{tabular}


TABLE VIII. Same as Table VII, but for case 2 .

\begin{tabular}{|c|c|c|c|c|c|c|c|c|c|c|c|c|}
\hline & & \multicolumn{5}{|c|}{ EOMS 2} & \multicolumn{5}{|c|}{ HB 2} & \multirow[b]{2}{*}{ LQCD $[26,29]$} \\
\hline & & $\mathcal{O}\left(p^{2}\right)$ & $\mathcal{O}\left(p^{3}\right)_{\overline{3}}$ & $\mathcal{O}\left(p^{3}\right)_{6}$ & $\mathcal{O}\left(p^{3}\right)_{6^{*}}$ & $\mu_{\mathrm{tot}}$ & $\mathcal{O}\left(p^{2}\right)$ & $\mathcal{O}\left(p^{3}\right)_{\overline{3}}$ & $\mathcal{O}\left(p^{3}\right)_{6}$ & $\mathcal{O}\left(p^{3}\right)_{6^{*}}$ & $\mu_{\mathrm{tot}}$ & \\
\hline \multirow[t]{3}{*}{$B_{\overline{3}}$} & $\mu_{\Lambda_{c}^{+}}$ & 0.24 & $\cdots$ & $\ldots$ & $\cdots$ & 0.24 & 0.24 & $\ldots$ & $\ldots$ & $\ldots$ & 0.24 & $\cdots$ \\
\hline & $\mu_{\Xi_{c}^{+}}$ & 0.24 & $\cdots$ & $\cdots$ & $\cdots$ & 0.24 & 0.24 & $\cdots$ & $\cdots$ & $\ldots$ & 0.24 & $\cdots$ \\
\hline & $\mu_{\Xi_{c}^{0}}^{0}$ & 0.19 & $\cdots$ & $\cdots$ & $\cdots$ & 0.19 & 0.19 & $\cdots$ & $\cdots$ & $\cdots$ & 0.19 & $\cdots$ \\
\hline \multirow[t]{6}{*}{$B_{6}$} & $\mu_{\Sigma_{c}^{++}}$ & 1.69 & $\cdots$ & -0.44 & 0.13 & 1.38 & 2.70 & $\cdots$ & -0.99 & 0.29 & 2.00 & $1.499(202)$ \\
\hline & $\mu_{\Sigma_{c}^{+}}$ & 0.32 & $\cdots$ & -0.09 & 0.04 & 0.27 & 0.72 & $\cdots$ & -0.35 & 0.09 & 0.46 & $\ldots$ \\
\hline & $\mu_{\Sigma_{c}^{0}}$ & -1.05 & $\cdots$ & 0.26 & -0.04 & -0.83 & -1.26 & $\cdots$ & 0.29 & -0.11 & -1.08 & $-0.875(103)$ \\
\hline & $\mu_{\Xi_{c}^{\prime+}}$ & 0.32 & $\cdots$ & 0.11 & -0.04 & 0.39 & 0.72 & $\ldots$ & -0.15 & 0.05 & 0.62 & $\ldots$ \\
\hline & $\mu_{\Xi_{c}^{\prime \prime}}$ & -1.05 & $\cdots$ & 0.38 & -0.10 & -0.77 & -1.26 & $\ldots$ & 0.49 & -0.14 & -0.91 & $\ldots$ \\
\hline & $\mu_{\Omega_{c}^{0}}^{0}$ & -1.05 & $\cdots$ & 0.51 & -0.15 & -0.69 & -1.26 & $\cdots$ & 0.70 & -0.18 & -0.74 & $-0.667(96)$ \\
\hline
\end{tabular}

shell (EOMS) scheme up to the next-to-leading order. Using the quark model and the heavy quark spin flavor symmetry to fix the low energy constants $g_{1,2,3,4}$, we determined the rest, i.e., $d_{2}, d_{3}, d_{5}$, and $d_{6}$, by fitting to the lattice QCD data. We compared our results with those of the heavy baryon (HB) ChPT and found that at NLO the lattice QCD quark mass dependent data can be better described by the EOMS BChPT, consistent with previous studies, though only marginally.

In addition, we notice that for the antitriplet baryons, the contributions of intermediate 6 and $6^{*}$ baryons exhibit a nice cancellation in the HB ChPT, while such a cancellation was not there in the EOMS BChPT because of the appearance of higher order relativistic corrections. The better agreement of our $\mathcal{O}\left(p^{3}\right)$ EOMS results with those of HB ChPT up to $\mathcal{O}\left(p^{4}\right)$ of Ref. [24] might indeed indicate that these corrections are relatively important.

We have treated the intermediate $6^{*}$ baryons using the so-called "consistent" coupling scheme and found indeed that it can provide a better description of the lattice QCD data compared to the conventional coupling scheme, but only moderately, different from the case of the ground-state octet baryons.

Compared with the results of other approaches, our predicted magnetic moments for both the antitriplet and the sextet baryons are relatively small in absolute values. Apparently, this is tied to the lattice QCD data of
Refs. [26-29], to which we fitted to fix the unknown low energy constants. It is not clear how to understand such a pattern at present. We hope that future lattice QCD and/or experimental studies can help us gain more insight into these important quantities and to better understand the nature of the singly charmed baryons. In addition, once more data become available, it might be worthwhile to perform a more rigorous study of the theoretical uncertainties, while the present work should be better seen as an exploration.

\section{ACKNOWLEDGMENTS}

R.X. S. thanks Jun-Xu Lu, Xiu-Lei Ren, and Jorge Martin Camalich for useful discussions. This work is partly supported by the National Natural Science Foundation of China under Grants No. 11522539, No. 11735003, and the fundamental Research Funds for the Central Universities.

\section{APPENDIX: LOOP FUNCTIONS}

Here, we provide the pertinent loop functions appearing in Eq. (7), corresponding to those Feynman diagrams of Fig. 1, regularized with the $\widetilde{\mathrm{MS}}$ scheme and with the $\mathrm{PCB}$ terms removed. All the loop functions, contributing either to the antitriplet or to the sextet singly charmed baryons, can be reduced to four common ones. More specifically, for the antitriplet baryons, we have

$$
\begin{array}{ll}
H_{B_{\overline{3}}}^{(b)}\left(\delta_{1}, m_{\phi}\right)=H^{(b)}\left(m_{\overline{3}}, \delta_{1}, m_{\phi}\right), & H_{B_{\overline{3}}}^{(c)}\left(\delta_{2}, m_{\phi}\right)=H^{(c)}\left(m_{\overline{3}}, \delta_{2}, m_{\phi}\right), \\
H_{B_{\overline{3}}}^{(d)}\left(\delta_{1}, m_{\phi}\right)=H^{(d)}\left(m_{\overline{3}}, \delta_{1}, m_{\phi}\right), & H_{B_{\overline{3}}}^{(e)}\left(\delta_{2}, m_{\phi}\right)=H^{(e)}\left(m_{\overline{3}}, \delta_{2}, m_{\phi}\right),
\end{array}
$$

where the functions $H^{(b, c, d, e)}$ are given below. For the sextet baryons, we have

$$
\begin{aligned}
H_{B_{6}}^{(b)}\left(0, m_{\phi}\right)=H^{(b)}\left(m_{6}, 0, m_{\phi}\right), & H_{B_{6}}^{(d)}\left(0, m_{\phi}\right)=H^{(d)}\left(m_{6}, 0, m_{\phi}\right), \\
H_{B_{6}}^{(b)}\left(\delta_{1}, m_{\phi}\right)=H^{(b)}\left(m_{6},-\delta_{1}, m_{\phi}\right), & H_{B_{6}}^{(d)}\left(\delta_{1}, m_{\phi}\right)=H^{(d)}\left(m_{6},-\delta_{1}, m_{\phi}\right), \\
H_{B_{6}}^{(c)}\left(\delta_{3}, m_{\phi}\right)=H^{(c)}\left(m_{6}, \delta_{3}, m_{\phi}\right), & H_{B_{6}}^{(e)}\left(\delta_{3}, m_{\phi}\right)=H^{(e)}\left(m_{6}, \delta_{3}, m_{\phi}\right) .
\end{aligned}
$$


The two common functions $H^{(b, d)}$ are,

$$
\begin{aligned}
H^{(b)}\left(m_{B}, \pm \delta, m_{\phi}\right)= & \frac{\left(m_{B}+m_{i}\right)^{2}}{16 \pi^{2} m_{B}^{4}}\left[\left(m_{B}-m_{i}\right) m_{i}^{3} \log \left(\frac{\left(m_{B}^{2}-m_{i}^{2}\right)^{2}}{m_{i}^{2} m_{\phi}^{2}}\right)-m_{\phi}^{2}\left(2 m_{B}^{2}+\left(m_{i} m_{B}+M^{2}\right) \log \left(\frac{m_{i}^{2}}{m_{\phi}^{2}}\right)\right)\right. \\
+ & \frac{2 \sqrt{\left(m_{B}-m_{i}\right)^{2}-m_{\phi}^{2}}\left(m_{\phi}^{2}\left(m_{i} m_{B}-M^{2}\right)-m_{i}^{3}\left(m_{B}+m_{i}\right)\right)}{\sqrt{\left(m_{B}+m_{i}\right)^{2}-m_{\phi}^{2}}} \\
\times & \log \left( \pm \sqrt{\left.\left.\frac{M^{2}+3 m_{i}^{2}-\lambda^{1 / 2}\left(m_{B}^{2}, m_{\phi}^{2}, m_{i}^{2}\right)}{M^{2}+3 m_{i}^{2}+\lambda^{1 / 2}\left(m_{B}^{2}, m_{\phi}^{2}, m_{i}^{2}\right)}\right)\right],}\right. \\
H^{(d)}\left(m_{B}, \pm \delta, m_{\phi}\right)= & \frac{\left(m_{B}+m_{i}\right)^{2}}{16 \pi^{2} m_{B}^{4}}\left[\frac{\left(m_{B}^{2}-m_{i}^{2}\right)^{2} \log \left(\frac{m_{i}^{2} m_{\phi}^{2}}{\left(m_{B}^{2}-m_{i}^{2}\right)^{2}}\right)}{1+m_{B} / m_{i}}-m_{\phi}^{2}\left(2 m_{B}^{2}+\left(M^{2}+m_{B}^{2}+m_{B} m_{i}\right) \log \left(\frac{m_{i}^{2}}{m_{\phi}^{2}}\right)\right)\right. \\
& \left.-\frac{2\left(\left(m_{B}-m_{i}\right)^{2}-m_{\phi}^{2}\right)\left(\frac{m_{i}\left(m_{B}^{2}-m_{i}^{2}\right)^{2}}{m_{B}-m_{i}}-m_{\phi}^{4}+m_{i}\left(m_{B}+2 m_{i}\right) m_{\phi}^{2}\right)}{\sqrt{-\lambda\left(m_{B}^{2}, m_{\phi}^{2}, m_{i}^{2}\right)}} \cos ^{-1}\left(\frac{M^{2}+3 m_{i}^{2}}{2 m_{i} m_{\phi}}\right)\right],
\end{aligned}
$$

with

$$
\lambda(a, b, c)=a^{2}+b^{2}+c^{2}-2 a b-2 a c-2 b c, \quad M^{2}=m_{\phi}^{2}-2 m_{i}^{2}-m_{B}^{2}, \quad m_{i}=m_{B}+\delta, \quad \delta>0 .
$$

It should be noted that $H^{(b)}\left(m_{B}, 0, m_{\phi}\right)$ and $H^{(d)}\left(m_{B}, 0, m_{\phi}\right)$ can easily be obtained by taking the limit of $\delta \rightarrow 0$ from Eqs. (A3), (A4). On the other hand, $H^{(c)}\left(m_{B}, \delta, m_{\phi}\right)$ and $H^{(e)}\left(m_{B}, \delta, m_{\phi}\right)$ correspond to $\frac{m_{B}+\delta}{12 \pi^{2}} \cdot\left(H^{(a)}-H_{\mathrm{PC}}^{(a)}\right)$ and $\frac{m_{B}+\delta}{12 \pi^{2}} \cdot\left(H^{(b)}-H_{\mathrm{PC}}^{(b)}\right)$, which are explicitly given in the Appendix of Ref. [42]. Here, the PCB terms $H_{\mathrm{PC}}^{(a)}$ and $H_{\mathrm{PC}}^{(b)}$ can be derived by expanding $m_{\phi}$ up to $\mathcal{O}\left(p^{0}\right)$. Note that in removing the PCB terms, we have kept the $\delta$ corrections up to all orders [42].

[1] M. Tanabashi et al. (Particle Data Group), Phys. Rev. D 98, 030001 (2018).

[2] R. Aaij et al. (LHCb Collaboration), J. High Energy Phys. 05 (2017) 030.

[3] R. Aaij et al. (LHCb Collaboration), Phys. Rev. Lett. 118, 182001 (2017).

[4] R. Aaij et al. (LHCb Collaboration), Phys. Rev. Lett. 119, 112001 (2017).

[5] H. X. Chen, W. Chen, X. Liu, and S. L. Zhu, Phys. Rep. 639, 1 (2016).

[6] H. X. Chen, W. Chen, X. Liu, Y. R. Liu, and S. L. Zhu, Rep. Prog. Phys. 80, 076201 (2017).

[7] F. K. Guo, C. Hanhart, U. G. Meißner, Q. Wang, Q. Zhao, and B. S. Zou, Rev. Mod. Phys. 90, 015004 (2018).

[8] A. Esposito, A. Pilloni, and A. D. Polosa, Phys. Rep. 668, 1 (2017).
[9] R. F. Lebed, R. E. Mitchell, and E. S. Swanson, Prog. Part. Nucl. Phys. 93, 143 (2017).

[10] G. Eichmann, H. Sanchis-Alepuz, R. Williams, R. Alkofer, and C. S. Fischer, Prog. Part. Nucl. Phys. 91, 1 (2016).

[11] S. L. Olsen, T. Skwarnicki, and D. Zieminska, Rev. Mod. Phys. 90, 015003 (2018).

[12] A. Ali, J. S. Lange, and S. Stone, Prog. Part. Nucl. Phys. 97, 123 (2017).

[13] N. Barik and M. Das, Phys. Rev. D 28, 2823 (1983).

[14] B. Julia-Diaz and D. O. Riska, Nucl. Phys. A739, 69 (2004).

[15] S. Kumar, R. Dhir, and R. C. Verma, J. Phys. G 31, 141 (2005).

[16] A. Faessler, T. Gutsche, M. A. Ivanov, J. G. Korner, V. E. Lyubovitskij, D. Nicmorus, and K. Pumsa-ard, Phys. Rev. D 73, 094013 (2006). 
[17] B. Patel, A. K. Rai, and P. C. Vinodkumar, J. Phys. G 35, 065001 (2008); J. Phys. Conf. Ser. 110, 122010 (2008).

[18] N. Sharma, H. Dahiya, P. K. Chatley, and M. Gupta, Phys. Rev. D 81, 073001 (2010).

[19] A. Bernotas and V. Simonis, arXiv:1209.2900.

[20] G. S. Yang and H. C. Kim, Phys. Lett. B 781, 601 (2018).

[21] J. Y. Kim and H. C. Kim, Phys. Rev. D 97, 114009 (2018).

[22] S. L. Zhu, W. Y. P. Hwang, and Z. S. Yang, Phys. Rev. D 56, 7273 (1997).

[23] T. M. Aliev, T. Barakat, and M. Savci, Phys. Rev. D 91, 116008 (2015).

[24] G. J. Wang, L. Meng, H. S. Li, Z. W. Liu, and S. L. Zhu, Phys. Rev. D 98, 054026 (2018).

[25] G. J. Wang, L. Meng, and S. L. Zhu, Phys. Rev. D 99, 034021 (2019).

[26] K. U. Can, G. Erkol, B. Isildak, M. Oka, and T. T. Takahashi, J. High Energy Phys. 05 (2014) 125.

[27] K. U. Can, G. Erkol, M. Oka, and T. T. Takahashi, Phys. Rev. D 92, 114515 (2015).

[28] H. Bahtiyar, K. U. Can, G. Erkol, M. Oka, and T. T. Takahashi, Phys. Lett. B 772, 121 (2017).

[29] H. Bahtiyar, K. U. Can, G. Erkol, and M. Oka, Phys. Lett. B 747, 281 (2015).

[30] X.-L. Ren, L. S. Geng, J. M. Camalich, J. Meng, and H. Toki, J. High Energy Phys. 12 (2012) 073.

[31] Y. Xiao, X. L. Ren, J. X. Lu, L. S. Geng, and U. G. Meißner, Eur. Phys. J. C 78, 489 (2018).
[32] S. Weinberg, Physica (Amsterdam) 96A, 327 (1979).

[33] E. E. Jenkins and A. V. Manohar, Phys. Lett. B 255, 558 (1991).

[34] V. Bernard, N. Kaiser, and U. G. Meissner, Int. J. Mod. Phys. E 04, 193 (1995).

[35] T. Becher and H. Leutwyler, Eur. Phys. J. C 9, 643 (1999).

[36] T. Fuchs, J. Gegelia, G. Japaridze, and S. Scherer, Phys. Rev. D 68, 056005 (2003).

[37] L. Geng, Front. Phys. (Beijing) 8, 328 (2013).

[38] M. Z. Liu, Y. Xiao, and L. S. Geng, Phys. Rev. D 98, 014040 (2018).

[39] A. N. H. Blin, Z. F. Sun, and M. J. Vicente Vacas, Phys. Rev. D 98, 054025 (2018).

[40] V. Pascalutsa, Phys. Rev. D 58, 096002 (1998).

[41] V. Pascalutsa and R. Timmermans, Phys. Rev. C 60, 042201 (1999).

[42] L. S. Geng, J. M. Camalich, and M. J. V. Vacas, Phys. Lett. B 676, 63 (2009).

[43] N. Jiang, X. L. Chen, and S. L. Zhu, Phys. Rev. D 92, 054017 (2015).

[44] N. Jiang, X. L. Chen, and S. L. Zhu, Phys. Rev. D 90, 074011 (2014).

[45] W. Detmold, C. J. D. Lin, and S. Meinel, Phys. Rev. D 85, 114508 (2012).

[46] W. Detmold, C.-J. D. Lin, and S. Meinel, Phys. Rev. Lett. 108, 172003 (2012). 